Proceedings of the Edinburgh Mathematical Society (2002) 45, 589-600 (C)

DOI:10.1017/S0013091501000943 Printed in the United Kingdom

\title{
ON A GAMMA FUNCTION INEQUALITY OF GAUTSCHI
}

\author{
HORST ALZER
}

Morsbacher Str. 10, D-51545 Waldbröl, Germany

(alzer@wmax03.mathematik.uni-wuerzburg.de)

(Received 22 October 2001)

Abstract We prove the following.

(1) The inequalities

$$
\left(2-\frac{1}{\Gamma(x)}\right)^{a}+\left(2-\frac{1}{\Gamma(1 / x)}\right)^{a} \leqslant 2 \leqslant\left(2-\frac{1}{\Gamma(x)}\right)^{b}+\left(2-\frac{1}{\Gamma(1 / x)}\right)^{b}
$$

hold for all $x>0$ if and only if

$$
-1.20464 \ldots=2+\frac{1}{\gamma}-\frac{1}{6}\left(\frac{\pi}{\gamma}\right)^{2} \leqslant a \leqslant 0 \leqslant b .
$$

(2) For all real numbers $x \in(0,1]$ we have

$$
x^{\alpha} \leqslant \frac{1}{2}\left(\frac{1}{\Gamma(x)}+\frac{1}{\Gamma(1 / x)}\right) \leqslant x^{\beta},
$$

with the best possible constants

$$
\alpha=1.32176 \ldots \text { and } \beta=0 .
$$

These theorems extend and complement a result of Gautschi (from 1974), who proved that for all $x>0$ the harmonic mean of $\Gamma(x)$ and $\Gamma(1 / x)$ is greater than or equal to 1.

Keywords: gamma function; inequalities; mean values

AMS 2000 Mathematics subject classification: Primary 33B15; 26D15

\section{Introduction}

In 1974, Gautschi [6] published the following interesting inequality for Euler's gamma function:

$$
\Gamma(x)=\int_{0}^{\infty} \mathrm{e}^{-t} t^{x-1} \mathrm{~d} t \quad(x>0) .
$$

For all $x>0$ we have

$$
\frac{1}{\Gamma(x)}+\frac{1}{\Gamma(1 / x)} \leqslant 2 .
$$

Inequality (1.1) states that the harmonic mean of $\Gamma(x)$ and $\Gamma(1 / x)$ is greater than or equal to 1 . This result has found the attention of several mathematicians, who proved various extensions, refinements and companions of (1.1). 
The power mean of order $t \in \mathbb{R}$ of the positive real numbers $x$ and $y$ is defined by

$$
M_{t}(x, y)=\left(\frac{1}{2}\left(x^{t}+y^{t}\right)\right)^{1 / t} \quad(t \neq 0), \quad M_{0}(x, y)=\sqrt{x y} .
$$

The most important properties of these and other mean values are given in the monograph [5].

Using the notation of power means, we can write (1.1) as

$$
1 \leqslant M_{-1}(\Gamma(x), \Gamma(1 / x)) \quad(x>0) .
$$

An extension of (1.2) can be found in [3]. The inequality

$$
1 \leqslant M_{t}(\Gamma(x), \Gamma(1 / x))
$$

holds for all $x>0$ if and only if $t \geqslant(1 / \gamma)-\left(\pi^{2} /\left(6 \gamma^{2}\right)\right)=-3.20464 \ldots$ Here, $\gamma$ denotes Euler's constant.

Since the power mean is increasing with respect to its order (see $[\mathbf{5}, \mathrm{p} .159])$, we obtain from $(1.2)$

$$
1 \leqslant \Gamma(x) \Gamma(1 / x) \quad(x>0),
$$

which was also proved by Kairies [10]. Laforgia and Sismondi [11] provided a counterpart to $(1.4)$ :

$$
\frac{1}{\Gamma(1+\lambda)} \leqslant\left[\frac{\Gamma(1+x) \Gamma(1+1 / x)}{\Gamma(\lambda+x) \Gamma(\lambda+1 / x)}\right]^{1 / 2} \quad(x>0 ; 0<\lambda<1) .
$$

If $\lambda>1$, then the reversed inequality is valid. The following double inequality was recently published by Giordano and Laforgia $[\mathbf{9}]$ :

$$
\frac{1}{2} \leqslant \frac{\Gamma(1+x) \Gamma(1+1 / x)}{\Gamma(1+x+1 / x)}<1 \quad(x>0) .
$$

In view of (1.1) it is tempting to conjecture that the inequality

$$
\sum_{k=1}^{n} \frac{1}{\Gamma\left(x_{k}\right)} \leqslant n
$$

holds for all positive real numbers $x_{k}(k=1, \ldots, n)$ satisfying $\prod_{k=1}^{n} x_{k}=1$. This problem was attacked by Gautschi [7], who proved that if $n \geqslant 9$, then (1.7) is in general not true. Furthermore, he gave 'numerical evidence' [7, p. 282] that (1.7) is valid for all $n \leqslant 8$. But a proof for this conjecture is known only for $n=2$.

Lucht [12] established a generalization of (1.4). Let $c^{*}=0.46163 \ldots$ be the only positive solution of $c^{*} \psi\left(c^{*}\right)=-1$, where $\psi=\Gamma^{\prime} / \Gamma$ denotes the logarithmic derivative of the gamma function. Then we have for all positive real numbers $x_{k}$ and $p_{k}(k=1, \ldots, n)$ with $\sum_{k=1}^{n} p_{k}=1$ and $\prod_{k=1}^{n} x_{k}^{p_{k}} \geqslant c^{*}$ :

$$
\Gamma\left(\prod_{k=1}^{n} x_{k}^{p_{k}}\right) \leqslant \prod_{k=1}^{n}\left(\Gamma\left(x_{k}\right)\right)^{p_{k}} .
$$


A survey on gamma function inequalities and a detailed list of references on this subject can be found in $[\mathbf{8}, \S 5]$.

In this paper we continue the study of inequalities involving $\Gamma(x)$ and $\Gamma(1 / x)$. In $\S 3$ we determine all parameters $a$ and $b$ such that the double inequality

$$
\left(2-\frac{1}{\Gamma(x)}\right)^{a}+\left(2-\frac{1}{\Gamma(1 / x)}\right)^{a} \leqslant 2 \leqslant\left(2-\frac{1}{\Gamma(x)}\right)^{b}+\left(2-\frac{1}{\Gamma(1 / x)}\right)^{b}
$$

holds for all $x>0$. We remark that the right-hand side of (1.8) with $b=1$ is equivalent to inequality (1.1).

Let

$$
G(x)=\frac{1}{2}\left(\frac{1}{\Gamma(x)}+\frac{1}{\Gamma(1 / x)}\right) .
$$

We have $G(1)=1$ and $\lim _{x \rightarrow 0} G(x)=0$, which implies that the constant bounds in

$$
0 \leqslant G(x) \leqslant 1 \quad(0<x \leqslant 1)
$$

cannot be improved. Numerous computer calculations suggested that the function $G$ can be approximated on the unit interval by powers of $x$. More precisely, these experiments led to the conjecture that for all $x \in(0,1]$ the value $x^{4 / 3}$ is a lower bound for $G(x)$. In $\S 3$ we prove that this is true. We determine the smallest number $\alpha$ (that is, we present exactly five places of decimals of the numerical value of $\alpha$ ) and we provide the largest number $\beta$ such that the inequalities

$$
x^{\alpha} \leqslant G(x) \leqslant x^{\beta}
$$

are valid for all $x \in(0,1]$.

The numerical values given in $\S \S 2$ and 3 have been found by computer computations carried out by MAPLE V, release 5.1.

\section{Lemmas}

In this section we collect several lemmas that we need to prove our main results. Throughout, we denote by $c=1.46163 \ldots$ the only positive zero of $\psi$. Furthermore, let $r=0.14$ and $s=0.215$.

Lemma 2.1. For all integers $n \geqslant 1$ and for all real numbers $x>0$ we have

$$
(-1)^{n+1} \psi^{(n)}(x)=n ! \sum_{k=0}^{\infty} \frac{1}{(x+k)^{n+1}} .
$$

The series representation (2.1) is given in [1, Equation 6.4.10].

Lemma 2.2. The function $\delta(x)=x \psi(x)$ is decreasing on $\left(0, c_{0}\right]$ and increasing on $\left[c_{0}, \infty\right)$, where $c_{0}=0.21609 \ldots$ is the unique positive root of $\psi(x)+x \psi^{\prime}(x)=0$. Furthermore, $\delta$ is convex on $(0, \infty)$. 
A proof of Lemma 2.2 is given in [2, Theorem 4], [7, Proposition 1] and [12, Satz 1]. The following two lemmas are proved in [4, Lemmas 1 and 2].

Lemma 2.3. Let $n \geqslant 1$ be an integer. The function $\phi_{n}(x)=x \psi^{(n+1)}(x) / \psi^{(n)}(x)$ is increasing on $(0, \infty)$.

Lemma 2.4. Let $\theta_{t, n}(x)=x^{t}\left|\psi^{(n)}(x)\right|$, where $t$ is a real number and $n \geqslant 1$ is an integer.

(i) If $t \leqslant n$, then $\theta_{t, n}$ is decreasing on $(0, \infty)$.

(ii) If $t \geqslant n+1$, then $\theta_{t, n}$ is increasing on $(0, \infty)$.

Lemma 2.5. Let $\lambda(x)=x \psi^{\prime}(x) / \psi(x)$.

(i) $\lambda$ is decreasing on $[r, s]$ and on $(c, \infty)$.

(ii) $\lambda$ is decreasing and concave on $(1 / c, c)$.

Proof. Part (ii) is proved in [3, Lemma 2]. To establish part (i) we define

$$
\lambda_{1}(x)=1+\phi_{1}(x)-\frac{\theta_{2,1}(x)}{\delta(x)},
$$

where $\delta, \phi_{1}, \theta_{2,1}$ are given in Lemmas 2.2-2.4. Since $\delta$ is negative on $[r, s]$, we get, for $x \in[r, s]$,

$$
\lambda_{1}(x) \geqslant 1+\phi_{1}(r)-\frac{\theta_{2,1}(r)}{\delta(s)}=0.017 \ldots
$$

From $\psi<0<\psi^{\prime}$ on $(0, c)$, we conclude that

$$
\lambda^{\prime}(x)=\frac{\psi^{\prime}(x)}{\psi(x)} \lambda_{1}(x)<0 \quad \text { for } x \in[r, s] .
$$

Let $x>c$. We have

$$
\lambda_{1}(x)=1+\phi_{1}(x)-\frac{\theta_{1,1}(x)}{\psi(x)} .
$$

The function $\phi_{1}$ is increasing on $(c, \infty)$, whereas $\theta_{1,1}$ and $1 / \psi$ are decreasing and positive on $(c, \infty)$. This implies that $\lambda_{1}$ is increasing on $(c, \infty)$. The limit relations

$$
\lim _{x \rightarrow \infty} x \psi^{\prime}(x)=-\lim _{x \rightarrow \infty} \frac{x \psi^{\prime \prime}(x)}{\psi^{\prime}(x)}=1, \quad \lim _{x \rightarrow \infty} \frac{x \psi^{\prime}(x)}{\psi(x)}=0
$$

(see [1, pp. 259, 260]) yield

$$
\lim _{x \rightarrow \infty} \lambda_{1}(x)=0
$$

Hence, $\lambda_{1}(x)<0$ for $x>c$. Since $\psi$ and $\psi^{\prime}$ are positive on $(c, \infty)$, we obtain $\lambda^{\prime}(x)<0$ for $x>c$.

Lemma 2.6. The function $\mu(x)=x \psi(x) /[2 \Gamma(x)-1]$ is strictly increasing on $[1, c)$ and strictly convex on $(1 / c, c)$. 
Proof. Let $\delta$ and $\lambda$ be the functions defined in Lemmas 2.2 and 2.5. Since $-\Gamma^{\prime}$ and $-\delta$ are positive and decreasing on $[1, c)$ we obtain

$$
2 \Gamma^{\prime}(x) \delta(x) \leqslant 2 \Gamma^{\prime}(1) \delta(1)=0.666 \ldots \quad(1 \leqslant x<c) .
$$

The function $\chi(x)=2 \Gamma(x)-1$ is positive and decreasing on $(0, c]$. Furthermore, $\delta^{\prime}$ is increasing and non-negative on $[1, c)$. Thus, we get

$$
\delta^{\prime}(x) \chi(x) \geqslant \delta^{\prime}(1) \chi(c)=0.823 \ldots \quad(1 \leqslant x<c) .
$$

Using (2.2) and (2.3) we obtain

$$
\mu^{\prime}(x)(\chi(x))^{2}=\delta^{\prime}(x) \chi(x)-2 \Gamma^{\prime}(x) \delta(x) \geqslant 0.15 \text { for } x \in[1, c) .
$$

We have

$$
\mu^{\prime \prime}(x)(\chi(x))^{2}=\left[2 \psi^{\prime}(x)+x \psi^{\prime \prime}(x)\right] \chi(x)-2 \Lambda(x)(\psi(x))^{2} \Gamma(x),
$$

where

$$
\Lambda(x)=2+3 \lambda(x)-\delta(x) \xi(x) \quad \text { and } \quad \xi(x)=1+\frac{1}{\Gamma(x)-\frac{1}{2}} .
$$

Applying (2.1) we get

$$
2 \psi^{\prime}(x)+x \psi^{\prime \prime}(x)=2 \sum_{k=1}^{\infty} \frac{k}{(x+k)^{3}}>0 \quad \text { for } x>0 .
$$

Since $\xi$ is positive and increasing on $(0, c)$, we obtain from Lemmas 2.2 and 2.5 for $1 / c \leqslant a \leqslant x<b \leqslant c$ :

$$
\Lambda(x) \leqslant 2+3 \lambda(a)-\delta(a) \xi(b)=\Omega(a, b), \quad \text { say. }
$$

We have

$$
\Omega(1 / c, 1)=-0.168 \ldots \quad \text { and } \quad \Omega(1, c)=-4.475 \ldots
$$

This implies

$$
\Lambda(x)<0 \quad \text { for } x \in(1 / c, c) .
$$

From (2.4)-(2.6) we conclude that $\mu^{\prime \prime}$ is positive on $(1 / c, c)$.

Lemma 2.7. The function $\nu(x)=\psi^{\prime}(x) / \psi(x)$ is increasing on $[r, s]$.

Proof. Let $\phi_{1}$ and $\lambda$ be defined as in Lemmas 2.3 and 2.5. Then we have

$$
\frac{x \psi(x)}{\psi^{\prime}(x)} \nu^{\prime}(x)=\phi_{1}(x)-\lambda(x)=\nu_{1}(x), \quad \text { say } .
$$

Since $\nu_{1}$ is increasing on $[r, s]$, we get

$$
\nu_{1}(x) \leqslant \nu_{1}(s)=-0.905 \ldots \text { for } x \in[r, s] .
$$

This implies that $\nu^{\prime}$ is positive on $[r, s]$. 
Lemma 2.8. The function $\rho(x)=\psi^{\prime \prime}(x) /(\psi(x))^{2}$ is increasing on $[r, s]$.

Proof. If $\phi_{2}$ and $\lambda$ are the functions given in Lemmas 2.3 and 2.5, then we get

$$
\frac{x(\psi(x))^{2}}{\psi^{\prime \prime}(x)} \rho^{\prime}(x)=\phi_{2}(x)-2 \lambda(x)=\rho_{1}(x), \quad \text { say } .
$$

Since

$$
\rho_{1}(x) \leqslant \rho_{1}(s)=-0.983 \ldots \text { for } x \in[r, s],
$$

we conclude that $\rho^{\prime}$ is positive on $[r, s]$.

Lemma 2.9. The function $\omega(x)=\left[(\psi(x))^{2}-\psi^{\prime}(x)\right] / \Gamma(x)$ is decreasing on $[r, s]$.

Proof. Let $\nu$ and $\rho$ be the functions defined in Lemmas 2.7 and 2.8. We have

$$
\frac{\Gamma(x)}{(\psi(x))^{2}} \omega^{\prime}(x)=3 \nu(x)-\rho(x)-\psi(x)=\omega_{1}(x), \quad \text { say. }
$$

Let $r \leqslant a \leqslant x \leqslant b \leqslant s$. Then we obtain

$$
\omega_{1}(x) \leqslant 3 \nu(b)-\rho(a)-\psi(a)=\omega_{2}(a, b), \quad \text { say } .
$$

The numerical values

$$
\begin{array}{ll}
\omega_{2}(0.140,0.143)=-0.03 \ldots, & \omega_{2}(0.143,0.146)=-0.06 \ldots, \\
\omega_{2}(0.146,0.149)=-0.08 \ldots, & \omega_{2}(0.149,0.152)=-0.11 \ldots, \\
\omega_{2}(0.152,0.156)=-0.01 \ldots, & \omega_{2}(0.156,0.160)=-0.05 \ldots, \\
\omega_{2}(0.160,0.164)=-0.08 \ldots, & \omega_{2}(0.164,0.169)=-0.02 \ldots, \\
\omega_{2}(0.169,0.174)=-0.06 \ldots, & \omega_{2}(0.174,0.180)=-0.01 \ldots, \\
\omega_{2}(0.180,0.186)=-0.07 \ldots, & \omega_{2}(0.186,0.193)=-0.04 \ldots, \\
\omega_{2}(0.193,0.201)=-0.03 \ldots, & \omega_{2}(0.201,0.210)=-0.03 \ldots, \\
\omega_{2}(0.210,0.215)=-0.34 \ldots &
\end{array}
$$

reveal that $\omega_{1}(x)<0$ for $x \in[r, s]$. This implies that $\omega^{\prime}$ is also negative on $[r, s]$.

Lemma 2.10. The function $\sigma(x)=x^{3} / \Gamma(x)$ is decreasing on $[1 / s, 1 / r]$.

Proof. An application of Lemma 2.2 yields, for $x \in[1 / s, 1 / r]$,

$$
\frac{x}{\sigma(x)} \sigma^{\prime}(x)=3-\delta(x) \leqslant 3-\delta(1 / s)=-3.631 \ldots
$$

Thus, $\sigma^{\prime}$ is negative on $[1 / s, 1 / r]$.

Lemma 2.11. The function $\tau(x)=x(\psi(x))^{2}-2 \psi(x)-x \psi^{\prime}(x)$ is positive and increasing on $[1 / s, 1 / r]$. 
Proof. Let

$$
\tau_{1}(x)=\delta(x)-\lambda(x)-2,
$$

where $\delta$ and $\lambda$ are defined in Lemmas 2.2 and 2.5. Then we conclude that $\tau_{1}$ is increasing on $[1 / s, 1 / r]$ with $\tau_{1}(1 / s)=3.849 \ldots$ The representation $\tau=\psi \tau_{1}$ reveals that $\tau$ is the product of two functions, which are increasing and positive on $[1 / s, 1 / r]$.

\section{Main results}

We are now in a position to prove our main results. First, we present all real numbers $a$ and $b$ such that (1.8) is valid for all $x>0$.

Theorem 3.1. Let $a$ and $b$ be real numbers. The inequalities

$$
\left(2-\frac{1}{\Gamma(x)}\right)^{a}+\left(2-\frac{1}{\Gamma(1 / x)}\right)^{a} \leqslant 2 \leqslant\left(2-\frac{1}{\Gamma(x)}\right)^{b}+\left(2-\frac{1}{\Gamma(1 / x)}\right)^{b}
$$

hold for all positive real numbers $x$ if and only if

$$
-1.20464 \ldots=2+\frac{1}{\gamma}-\frac{1}{6}\left(\frac{\pi}{\gamma}\right)^{2} \leqslant a \leqslant 0 \leqslant b .
$$

Proof. Let $a b \neq 0$. First, we assume that (3.1) is valid for all $x>0$. If $x$ tends to $\infty$, then we get $2^{a+1} \leqslant 2 \leqslant 2^{b+1}$, which implies $a<0<b$. Furthermore, we have, for $x>0$,

$$
f_{a}(x)=2-\left(2-\frac{1}{\Gamma(x)}\right)^{a}-\left(2-\frac{1}{\Gamma(1 / x)}\right)^{a} \geqslant 0 .
$$

Since $f_{a}(1)=f_{a}^{\prime}(1)=0$, we obtain

$$
f_{a}^{\prime \prime}(1)=-\frac{1}{3} a\left[6 \gamma^{2} a+\pi^{2}-6 \gamma-12 \gamma^{2}\right] \geqslant 0 .
$$

This leads to

$$
a \geqslant 2+\frac{1}{\gamma}-\frac{1}{6}\left(\frac{\pi}{\gamma}\right)^{2}
$$

Let $u(x)=2-1 / \Gamma(x), v(x)=u(1 / x)$ and $a_{0}=2+(1 / \gamma)-\pi^{2} /\left(6 \gamma^{2}\right)$. We prove that the inequality

$$
1<M_{a_{0}}(u(x), v(x))
$$

is valid for $0<x \neq 1$. Let $a_{0} \leqslant a<0<b$. Using the monotonicity of the power mean we obtain, from (3.2),

$$
1<M_{a}(u(x), v(x)) \leqslant M_{b}(u(x), v(x)) \quad(0<x \neq 1) .
$$

This leads to (3.1) with ' $<$ ' instead of ' $\leqslant$ '.

We show that the function

$$
g(x)=(u(x))^{a_{0}}+(v(x))^{a_{0}}
$$


is strictly decreasing on $[1, \infty)$. Then we have

$$
g(x)<g(1)=2 \quad(x>1),
$$

so that the identity $g(x)=g(1 / x)$ yields $g(x)<2$ for $0<x \neq 1$. This proves (3.2).

Since $u$ and $v$ are strictly increasing on $[c, \infty)$ we conclude that $g$ is strictly decreasing on $[c, \infty)$. It remains to show that $g$ is also strictly decreasing on $(1, c)$. Let $x \in(1, c)$. A simple calculation yields that $g^{\prime}(x)<0$ is equivalent to $h(x)<0$, where

$$
h(x)=\left(a_{0}-1\right)[\log (u(x))-\log (v(x))]-\log \left(v^{\prime}(x)\right)+\log \left(-u^{\prime}(x)\right) .
$$

Next, we establish that $h$ is strictly decreasing on $(1, c)$. Differentiation gives

$$
x h^{\prime}(x)=\left(a_{0}-1\right)[\mu(x)+\mu(1 / x)]+\lambda(x)+\lambda(1 / x)-[\delta(x)+\delta(1 / x)]+2,
$$

where $\delta, \lambda$ and $\mu$ are defined in Lemmas 2.2, 2.5 and 2.6. We have $1 / c<1 / x<x<c$, so that the concavity of $-\delta, \lambda$ and $-\mu$ leads to

$$
\begin{gathered}
-[\delta(x)+\delta(1 / x)] \leqslant-2 \delta\left(\frac{1}{2}(x+1 / x)\right), \\
\lambda(x)+\lambda(1 / x) \leqslant 2 \lambda\left(\frac{1}{2}(x+1 / x)\right), \\
-[\mu(x)+\mu(1 / x)] \leqslant-2 \mu\left(\frac{1}{2}(x+1 / x)\right) .
\end{gathered}
$$

From (3.3)-(3.6) we get

$$
\frac{1}{2} x h^{\prime}(x) \leqslant\left(a_{0}-1\right) \mu\left(\frac{1}{2}(x+1 / x)\right)+\lambda\left(\frac{1}{2}(x+1 / x)\right)-\delta\left(\frac{1}{2}(x+1 / x)\right)+1 .
$$

We have $1<\frac{1}{2}(x+1 / x)<c$. The monotonicity of $\delta, \lambda, \mu$ and $\psi(1)=-\gamma, \psi^{\prime}(1)=\pi^{2} / 6$ yield

$$
\frac{1}{2} x h^{\prime}(x)<\left(a_{0}-1\right) \mu(1)+\lambda(1)-\delta(1)+1=\left(a_{0}-1\right) \psi(1)+\psi^{\prime}(1) / \psi(1)-\psi(1)+1=0 .
$$

Hence, $h$ is strictly decreasing on $(1, c)$, which implies $h(x)<h(1)=0$ for $x \in(1, c)$. Thus $g$ is strictly decreasing on $(1, c)$. This completes the proof of Theorem 3.1.

Remark 3.2. The proof of Theorem 3.1 reveals that if $a b \neq 0$, then the sign of equality holds in (3.1) if and only if $x=1$.

Remark 3.3. Let $a_{0}=2+(1 / \gamma)-\pi^{2} /\left(6 \gamma^{2}\right), a_{1}=a_{0}-2$ and $u(x)=2-1 / \Gamma(x)$. From (3.2) and (1.3) we obtain the power mean inequalities

$$
1 \leqslant M_{a_{0}}(u(x), u(1 / x)) \quad(x>0)
$$

and

$$
1 \leqslant M_{a_{1}}(\Gamma(x), \Gamma(1 / x)) \quad(x>0) .
$$

The function

$$
D(x)=M_{a_{1}}(\Gamma(x), \Gamma(1 / x))-M_{a_{0}}(u(x), u(1 / x))
$$

is positive for all sufficiently small $x$ and negative for all $x$, which are sufficiently close to 1 . Hence, (3.8) and (3.9) do not imply each other. 
Our second theorem provides the smallest constant $\alpha$ and the largest constant $\beta$ in (1.10). In particular, we obtain a refinement of the left-hand side of (1.9).

Theorem 3.4. For all real numbers $x \in(0,1]$ we have

$$
x^{\alpha} \leqslant \frac{1}{2}\left(\frac{1}{\Gamma(x)}+\frac{1}{\Gamma(1 / x)}\right) \leqslant x^{\beta},
$$

with the best possible constants

$$
\alpha=1.32176 \ldots \text { and } \beta=0 .
$$

More precisely, $\alpha$ satisfies the estimates $1.321767 \leqslant \alpha \leqslant 1.321769$.

Proof. Let $\alpha_{0}=1.321769$. First, we prove

$$
x^{\alpha_{0}} \leqslant \frac{1}{2}\left(\frac{1}{\Gamma(x)}+\frac{1}{\Gamma(1 / x)}\right) \text { for } x \in(0,1] .
$$

We consider four cases.

Case $1(x \in(0,0.14])$. Let

$$
f(x)=\log (\Gamma(x))+\alpha_{0} \log (x)+\log (2) .
$$

Applying Lemma 2.2 we get

$$
x f^{\prime}(x)=\delta(x)+\alpha_{0} \geqslant \delta(0.14)+\alpha_{0}=0.270 \ldots,
$$

which implies

$$
f(x) \leqslant f(0.14)=-0.005 \ldots
$$

This leads to

$$
x^{\alpha_{0}}<\frac{1}{2 \Gamma(x)}<\frac{1}{2}\left(\frac{1}{\Gamma(x)}+\frac{1}{\Gamma(1 / x)}\right) .
$$

Case $2(\boldsymbol{x} \in[\mathbf{0 . 1 4}, \mathbf{0 . 2 1 5}])$. Let $r=0.14$ and $s=0.215$, and

$$
g(x)=\frac{1}{\Gamma(x)}+\frac{1}{\Gamma(1 / x)}-2 x^{\alpha_{0}} .
$$

We prove that $g$ is strictly convex on $[r, s]$. Differentiation gives

$$
g^{\prime \prime}(x)=\omega(x)+\sigma(1 / x) \tau(1 / x)+\kappa(x),
$$

where $\omega, \sigma, \tau$ are defined in Lemmas 2.9-2.11, and

$$
\kappa(x)=2 \alpha_{0}\left(1-\alpha_{0}\right) x^{\alpha_{0}-2} .
$$

Let $r \leqslant a \leqslant x \leqslant b \leqslant s$. The monotonicity of $\omega, \sigma, \tau$ and $\kappa$ leads to

$$
g^{\prime \prime}(x) \geqslant \omega(b)+\sigma(1 / a) \tau(1 / b)+\kappa(a)=g_{1}(a, b), \quad \text { say. }
$$


Since

$$
g_{1}(r, 0.16)=2.930 \ldots \quad \text { and } \quad g_{1}(0.16, s)=4.615 \ldots,
$$

we conclude that $g^{\prime \prime}$ is positive on $[r, s]$, so that $g^{\prime}$ is strictly increasing on $[r, s]$. Let $y_{1}=0.157620$ and $y_{2}=0.157629$. We have $g^{\prime}\left(y_{1}\right)<0<g^{\prime}\left(y_{2}\right)$. This implies that there exists a number $x^{*} \in\left(y_{1}, y_{2}\right)$ such that $g^{\prime}$ is negative on $\left[r, x^{*}\right)$ and positive on $\left(x^{*}, s\right]$. Hence,

$$
g(x) \geqslant g\left(x^{*}\right) \text { for all } x \in[r, s] .
$$

The convexity of $g$ in combination with Taylor's Theorem yields

$$
g\left(x^{*}\right) \geqslant g\left(y_{2}\right)+\left(x^{*}-y_{2}\right) g^{\prime}\left(y_{2}\right) \geqslant g\left(y_{2}\right)+\left(y_{1}-y_{2}\right) g^{\prime}\left(y_{2}\right)=0.00000061 \ldots
$$

From (3.12) and (3.13) we conclude that $g$ is positive on $[r, s]$.

Case $3(x \in[0.215,0.42])$. Let

$$
h(x)=\frac{1}{\Gamma(x)}-2 x^{\alpha_{0}} .
$$

Differentiation yields

$$
-\Gamma(x) h^{\prime}(x)=\psi(x)+2 \alpha_{0} x^{\alpha_{0}-1} \Gamma(x)=u(x), \quad \text { say. }
$$

If $x \in[0.215,0.29]$, then we get

$$
u(x) \geqslant \psi(0.215)+2 \alpha_{0}(0.215)^{\alpha_{0}-1} \Gamma(0.29)=0.076 \ldots .
$$

Thus $h$ is decreasing and we obtain

$$
h(x)+\frac{1}{\Gamma(1 / x)} \geqslant h(0.29)+\frac{1}{\Gamma(1 / 0.215)}=0.002 \ldots
$$

If $x \in[0.29,0.42]$, then

$$
u(x) \geqslant \psi(0.29)+2 \alpha_{0}(0.29)^{\alpha_{0}-1} \Gamma(0.42)=0.117 \ldots .
$$

This implies

$$
h(x)+\frac{1}{\Gamma(1 / x)} \geqslant h(0.42)+\frac{1}{\Gamma(1 / 0.29)}=0.156 \ldots
$$

Case $4(x \in[\mathbf{0 . 4 2}, \mathbf{1}])$. We define

$$
v(x)=\log (\Gamma(x))+\log (\Gamma(1 / x))+2 \alpha_{0} \log (x) .
$$

Applying Lemma 2.2 we obtain

$$
x v^{\prime}(x)=\delta(x)-\delta(1 / x)+2 \alpha_{0} \geqslant \delta(0.42)-\delta(1 / 0.42)+2 \alpha_{0}=0.095 \ldots
$$

This implies

$$
v(x) \leqslant v(1)=0 .
$$


Inequality (3.14) and the geometric mean-harmonic mean inequality yield

$$
x^{\alpha_{0}} \leqslant[\Gamma(x) \Gamma(1 / x)]^{-1 / 2} \leqslant \frac{1}{2}\left(\frac{1}{\Gamma(x)}+\frac{1}{\Gamma(1 / x)}\right) .
$$

This completes the proof of (3.11).

Let

$$
\Delta(x)=\log \left[\frac{1}{2}\left(\frac{1}{\Gamma(x)}+\frac{1}{\Gamma(1 / x)}\right)\right] / \log (x) \quad(0<x<1) .
$$

Applying l'Hôpital's rule we obtain

$$
\Delta(1)=\lim _{x \rightarrow 1} \Delta(x)=0 .
$$

The inequalities (1.1) and (3.11) lead to

$$
0 \leqslant \Delta(x) \leqslant \alpha_{0} \text { for } x \in(0,1] .
$$

From (3.10) we conclude that the best possible constants $\alpha$ and $\beta$ are given by

$$
\alpha=\sup _{x \in(0,1]} \Delta(x) \text { and } \beta=\inf _{x \in(0,1]} \Delta(x) .
$$

Using (3.15) and (3.16) we obtain $\beta=0$. Furthermore, we have

$$
1.321767 \ldots=\Delta(0.157624) \leqslant \alpha \leqslant \alpha_{0}=1.321769 .
$$

Thus, $\alpha=1.32176 \ldots$ The proof of Theorem 3.4 is complete.

Acknowledgements. I thank the referee for his careful reading of the manuscript.

\section{References}

1. M. Abramowitz And I. A. Stegun (EDS), Handbook of mathematical functions with formulas, graphs and mathematical tables (Dover, New York, 1965).

2. H. Alzer, On some inequalities for the gamma and psi functions, Math. Computat. 66 (1997), 373-389.

3. H. Alzer, Inequalities for the gamma function, Proc. Am. Math. Soc. 128 (1999), 141147.

4. H. Alzer, Mean-value inequalities for the polygamma functions, Aequat. Math. 61 (2001), 151-161.

5. P. S. Bullen, D. S. Mitrinović and P. M. Vasić, Means and their inequalities (Reidel, Dordrecht, 1988).

6. W. Gautschi, A harmonic mean inequality for the gamma function, SIAM J. Math. Analysis 5 (1974), 278-281.

7. W. Gautschi, Some mean value inequalities for the gamma function, SIAM J. Math. Analysis 5 (1974), 282-292.

8. W. Gautschi, The incomplete gamma function since Tricomi, in Tricomi's ideas and contemporary applied mathematics, pp. 203-237, Atti Convegni Lincei, no. 147 (Accad. Naz. Lincei, Rome, 1998). 
9. C. Giordano And A. LAForgia, Inequalities and monotonicity properties for the gamma function, J. Comput. Appl. Math. 133 (2001), 387-396.

10. H. H. KAIRIES, An inequality for Krull solutions of a certain difference equation, in General inequalities, vol. 3 (ISNM, vol. 64), pp. 277-280 (Birkhäuser, Basel, 1983).

11. A. LAFORGIA AND S. Sismond, A geometric mean inequality for the gamma function, Boll. UMI 3 (1989), 339-342.

12. L. G. Lucht, Mittelwertungleichungen für Lösungen gewisser Differenzengleichungen, Aequat. Math. 39 (1990), 204-209. 